\title{
CASOS CONFIRMADOS DE TUBERCULOSE NO BRASIL, NA REGIÃO NORTE, NO ESTADO DO AMAPÁ E NO MUNICÍPIO DE MACAPÁ, ENTRE 2013 E 2017
}

\section{ARTIGO ORIGINAL}

JESUS, Michele da Cruz de ${ }^{1}$

SILVA, Lucas Facco ${ }^{2}$

FECURY, Amanda Alves ${ }^{3}$

OLIVEIRA, Euzébio de ${ }^{4}$

DENDASCK, Carla Viana ${ }^{5}$

DIAS, Cláudio Alberto Gellis de Mattos ${ }^{6}$

JESUS, Michele da Cruz de. Et al. Casos confirmados de tuberculose no Brasil, na Região Norte, no Estado do Amapá e no Município de Macapá, entre 2013 e 2017. Revista Científica Multidisciplinar Núcleo do Conhecimento. Ano 05, Ed. 05, Vol.

1 Discente do Curso de Educação Física da Universidade Federal do Amapá (UNIFAP).

${ }^{2}$ Discente do Curso de Medicina da Universidade Federal do Amapá (UNIFAP).

${ }^{3}$ Biomédica, Doutora em Doenças Topicais, Professora e pesquisadora do Curso de Medicina do Campus Macapá, Universidade Federal do Amapá (UNIFAP).

${ }^{4}$ Biólogo, Doutor em Doenças Topicais, Professor e pesquisador do Curso de Educação Física da, Universidade Federal do Pará (UFPA).

5 Teóloga, Doutora em Psicanálise, pesquisadora do Centro de Pesquisa e Estudos Avançados- CEPA.

${ }^{6}$ Biólogo, Doutor em Teoria e Pesquisa do Comportamento, Professor e pesquisador do Curso de Licenciatura em Química do Instituto de Ensino Básico, Técnico e tecnológico do Amapá (IFAP). 
01, pp. 144-154. Maio de 2020. ISSN: 2448-0959, Link de acesso: https://www.nucleodoconhecimento.com.br/saude/tuberculose-no-brasil, DOI: 10.32749/nucleodoconhecimento.com.br/saude/tuberculose-no-brasil

\section{RESUMO}

A tuberculose (TB) é uma doença infecciosa causada pela bactéria patogênica. $O$ bacilo é transmitido principalmente por meio do mecanismo da tosse, além do espirro e da fala, os quais encarregam-se de lançar gotículas de saliva. No Brasil 69.569 novos casos de tuberculose tiveram seu diagnóstico confirmado em 2017. No estado brasileiro do Amapá, ainda em 2017, houveram 237 novos casos de tuberculose notificados. O objetivo deste trabalho é comparar a porcentagem de casos confirmados de tuberculose no Brasil, na região Norte, no estado do Amapá e no município de Macapá, entre os anos de 2013 e 2017. Os dados foram coletados no site DATASUS. As coinfecções assim como o alcoolismo, uso de drogas, aglomerações urbanas e o estado nutricional do indivíduo também podem representar fatores que aumentam os casos de tuberculose. A vulnerabilidade social e a pouca atenção primária a saúde também favorecem o aumento desses números. Ter um Centro de Referência em Doenças Tropicais (CRDT), que também agrega o Centro de Testagem e Aconselhamento (CTA) e Assistência Domiciliar Terapêutica (ADT) auxilia na detecção de casos agilizando e controlando o tratamento de pacientes. Homens na faixa etária de trabalho são mais acometidos por conta da sua necessária interação social para o trabalho.

Palavras-chave: Tuberculose, epidemiologia, Região Norte, Amapá.

\section{INTRODUÇÃO}

A tuberculose (TB) é uma doença infecciosa causada pela bactéria patogênica Mycobacterium tuberculosis, conhecida também como Bacilo de Koch (BK) (MACEDO et al., 2017). 
Uma vez infectados, cerca de 5 a 10\% dos indivíduos apresentam a tuberculose ativa e, a depender do órgão acometido, podem manifestar uma série de sintomas, sendo a tosse o mais comum deles, evoluindo de seca a mucosa e, por vezes, associada a sangue, caracterizando hemoptise (tossir sangue). O quadro pode evoluir para dor torácica e, de uma forma menos comum, para dispneia (dificuldade em respirar), ambas ocasionadas por lesão e derrame da cavidade pleural. Febre e perda de peso também podem ser manifestações da doença (KOZAKEVICH e SILVA, 2015).

O bacilo é transmitido principalmente por meio do mecanismo da tosse, além do espirro e da fala, os quais encarregam-se de lançar gotículas de saliva (chamadas gotículas de Flügge) que, dependendo do peso delas, podem permanecer em suspensão aérea e possibilitar a infecção ao atingir bronquíolos e alvéolos pulmonares. Levando em consideração o grau de contato e a proximidade, estima-se que seja necessária uma exposição de cerca de 100 horas ou mais para que haja infecção interpessoal (KOZAKEVICH e SILVA, 2015).

A tuberculose (TB) possui cura por meio de tratamento eficaz, sendo esse realizado com a utilização de vários fármacos anti-TB (SILVA et al., 2018). De acordo com o PNCT (Programa Nacional de Controle da Tuberculose), do Ministério da Saúde, são quatro os medicamentos utilizados no esquema básico para o tratamento da tuberculose, de forma combinada, em adultos e adolescentes: Rifampicina $150 \mathrm{mg}$, Isoniazida $75 \mathrm{mg}$, Pirazinamida $400 \mathrm{mg}$ e Etambutol $275 \mathrm{mg}$. No caso da doença em crianças, retira-se o Etambutol $275 \mathrm{mg}$ do esquema, utilizando-se apenas os três primeiros (BRASIL, 2019).

De modo a buscar a prevenção contra a tuberculose, utiliza-se em muitos países, desde o século passado, a partir da década de 20, a vacina BCG em crianças de 0 a 4 anos. Ela previne as formas graves da doença, como as tuberculoses miliar (que se espalha no corpo podendo gerar lesões na pele) e meníngea (que pode atingir os tecidos que envolvem o cérebro, chamados meninges). No Brasil, a meta de vacinação preconizada pelo Programa Nacional de Imunizações (PNI) é de $90 \%$ das crianças de faixa etária menor que 1 ano de idade, a qual tem sido alcançada e extrapolada (BRASIL, 2018). Muito embora seja eficaz em crianças, a vacina BCG 
não demonstra a mesma eficácia preventiva em adultos, denotando que se faz necessária uma vacina eficiente para o amplo espectro de idades dos indivíduos (CARVALHO et al., 2018).

Estima-se que cerca de $25 \%$ da população mundial esteja infectada pelo Mycobacterium tuberculosis. Em 2017, por conta da tuberculose, aproximadamente 6,4 milhões de pessoas ficaram doentes, sendo que cerca de um quarto delas morreram (WHO, 2019).

No Brasil 69.569 novos casos de tuberculose tiveram seu diagnóstico confirmado em 2017, e, em 2016, houveram 4.426 óbitos por conta dessa doença (BRASIL, 2018).

Na região norte do país, em 2017, foram registrados 7653 casos novos de tuberculose, sendo a incidência de 42,7 em cada 100 mil habitantes e o coeficiente de mortalidade de 2,4 a cada 100 mil habitantes (BRASIL, 2018).

No estado brasileiro do Amapá, ainda em 2017, houveram 237 novos casos de tuberculose notificados, sendo que o coeficiente de incidência foi de 29,7 para cada 100 mil habitantes, enquanto o coeficiente de mortalidade foi de 1,4 em 100 mil habitantes (BRASIL, 2018).

\section{OBJETIVOS}

Comparar a porcentagem de casos confirmados de tuberculose no Brasil, na região Norte, no estado do Amapá e no município de Macapá, entre os anos de 2013 e 2017.

\section{MÉTODO}

Os dados foram coletados no site DATASUS[7]. A partir das seguintes etapas: A) Através do navegador de internet Chrome, acessou-se o link[8], na barra de funções do site passou -se o mouse sobre a opção "Acesso à informação" mantendo-o em cima, na aba que se abriu, arrastou-se o mouse até a opção "Informação de Saúde (TABNET)" e novamente se abriu outra aba na qual clicou-se em "Epidemiológicas de morbidade". Na página seguinte selecionou-se a opção "Casos de Tuberculose - 
Desde 2001 (SINAN)". Escolheu-se a única opção na página seguinte "Tuberculose desde 2001". Na mesma página, dentro da caixa de seleção "Abrangência geográfica" selecionou-se a opção "Brasil por Região, UF e Município". A) Na página que se seguiu, na caixa onde solicitava "Linha", selecionou a opção "Ano de Notificação"; na caixa que solicitava "Coluna", selecionou-se a opção "Região de Notificação"; e na caixa de "Períodos disponíveis", selecionou-se os anos de 2013 a 2017. B) Na página que se seguiu, na caixa onde solicitava "Linha", selecionou a opção "Ano de Notificação"; na caixa que solicitava "Coluna", selecionou-se a opção "UF de Notificação"; e na caixa de "Períodos disponíveis", selecionou-se os anos de 2013 a 2017. C) Na página que se seguiu, na caixa onde solicitava "Linha", selecionou a opção "Ano de Notificação"; na caixa que solicitava "Coluna", selecionou-se a opção "Capital de Notificação"; e na caixa de "Períodos disponíveis", selecionou-se os anos de 2013 a 2017. D) Na página que se seguiu, na caixa onde solicitava "Linha", selecionou a opção "Ano de Notificação"; na caixa que solicitava "Coluna", selecionouse a opção "Fx Etária"; e na caixa de "Períodos disponíveis", selecionou-se os anos de 2013 a 2017. E) Na página que se seguiu, na caixa onde solicitava "Linha", selecionou a opção "Ano de Notificação"; na caixa que solicitava "Coluna", selecionouse a opção "Escolaridade"; e na caixa de "Períodos disponíveis", selecionou-se os anos de 2013 a 2016. F) Na página que se seguiu, na caixa onde solicitava "Linha", selecionou a opção "Ano de Notificação"; na caixa que solicitava "Coluna", selecionouse a opção "Sexo"; e na caixa de "Períodos disponíveis", selecionou-se os anos de 2013 a 2016. G) Na página que se seguiu, na caixa onde solicitava "Linha", selecionou a opção "Ano de Notificação"; na caixa que solicitava "Coluna", selecionou-se a opção "Zona Residência"; e na caixa de "Períodos disponíveis", selecionou-se os anos de 2013 a 2017. A compilação dos dados foi feita dentro do aplicativo Excel, componente do pacote Office da Microsoft Corporation. A pesquisa bibliografia foi realizada em artigos científicos, utilizando-se para busca computadores do laboratório de informática do Instituto Federal de Educação, Ciência e Tecnologia do Amapá, Campus Macapá, situado na: Rodovia BR 210 KM 3, s/n - Bairro Brasil Novo. CEP: 68.909-398, Macapá, Amapá, Brasil. 


\section{RESULTADOS}

A figura 1 mostra a porcentagem de casos confirmados de tuberculose no Brasil entre 2013 e 2017. Entre os anos de 2013 e 2015 a porcentagem de casos diminuiu, voltando a aumentar em 2016 e 2017.

Figura 1 Mostra a porcentagem de casos confirmados de tuberculose no Brasil entre 2013 e 2017.

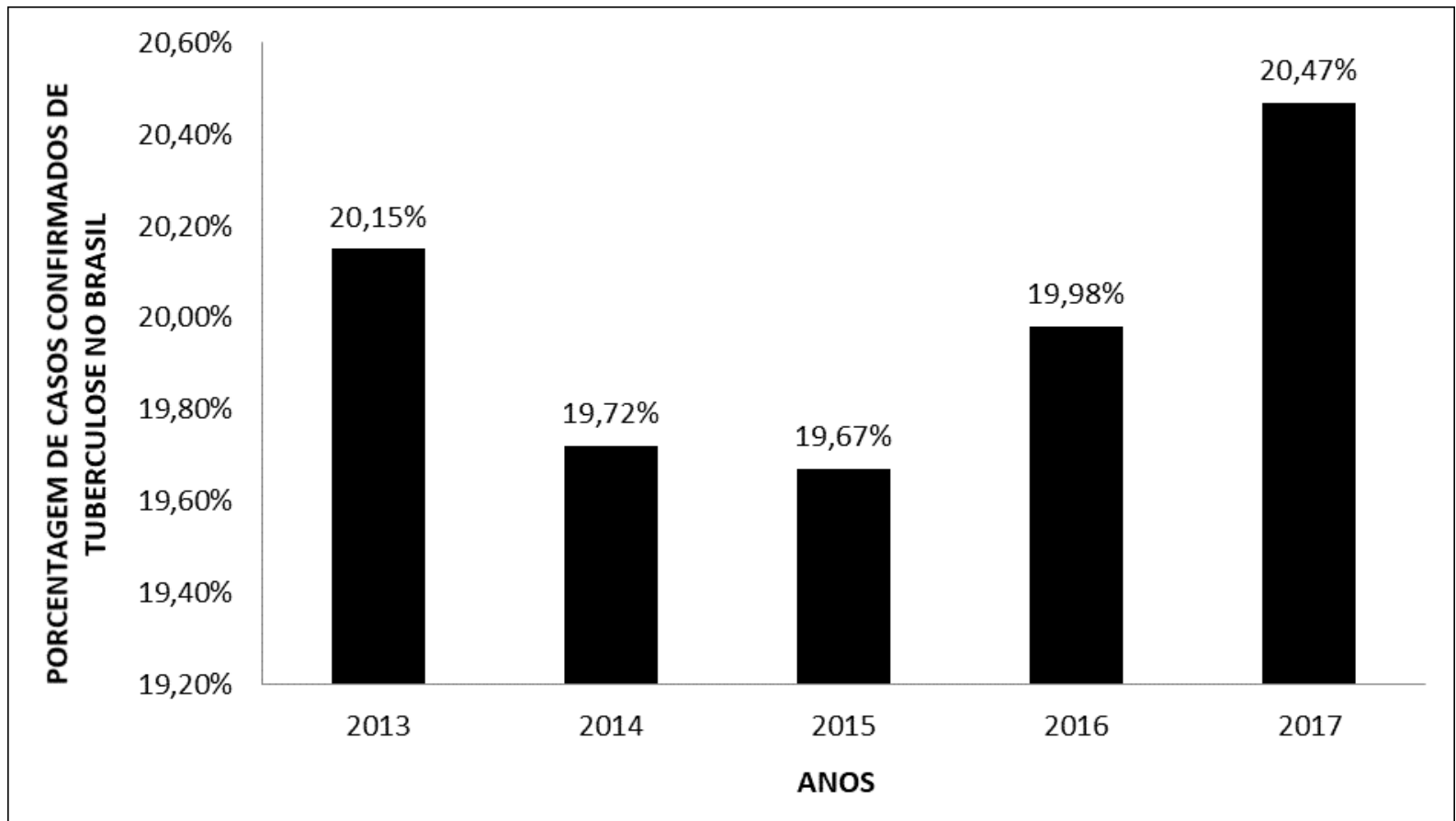

A figura 2 mostra a porcentagem de casos confirmados de tuberculose nas regiões do Brasil entre 2013 e 2017. A região Sudeste tem a maior porcentagem de casos confirmados, seguida pela região Nordeste e Sul, enquanto a menor está na CentroOeste e Norte. 
Figura 2 Mostra a porcentagem de casos confirmados de tuberculose nas regiões do Brasil entre 2013 e 2017.

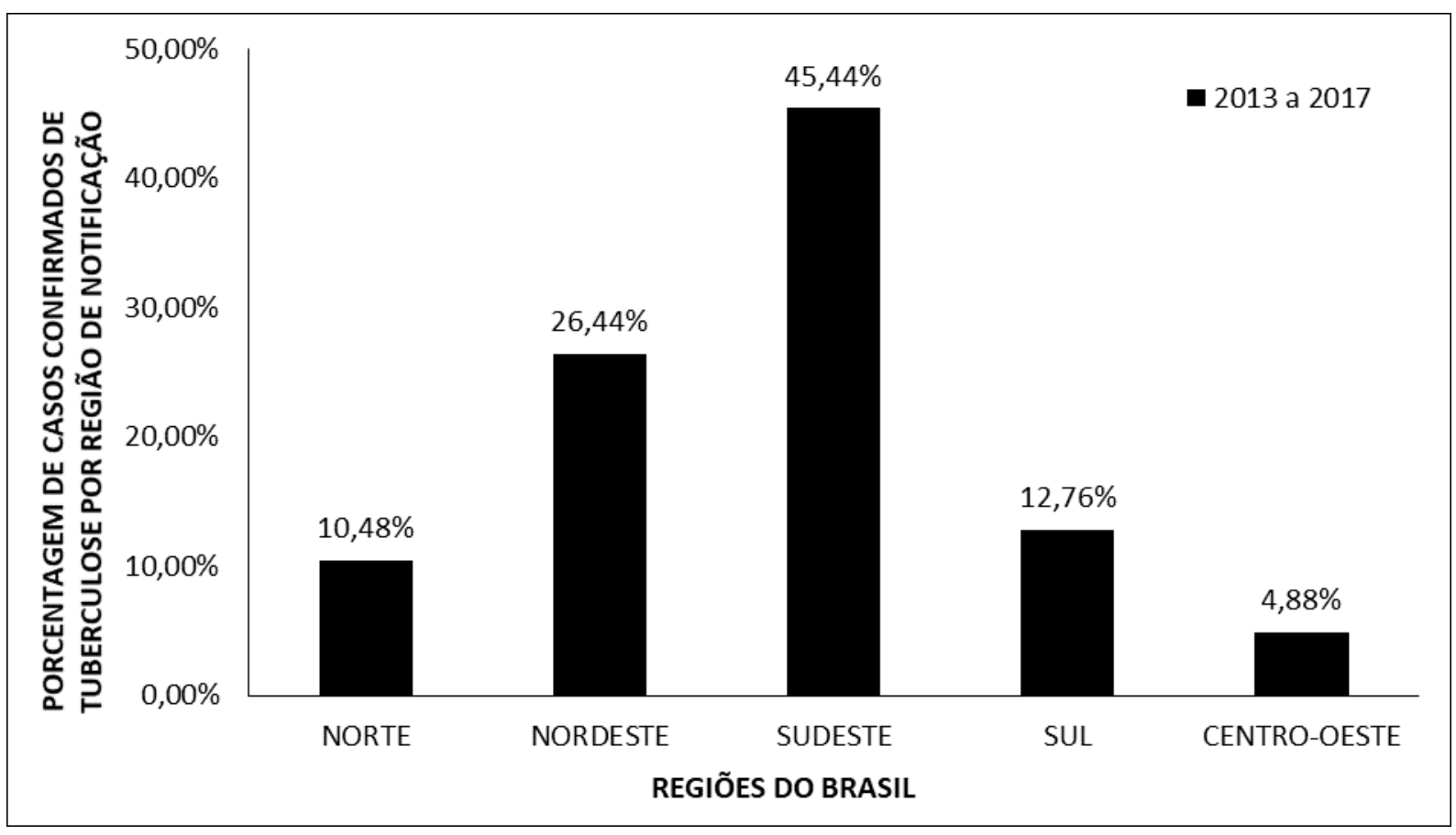

A figura 3 mostra a porcentagem de casos confirmados de tuberculose entre $2013 \mathrm{e}$ 2017 na região Norte do Brasil. As maiores porcentagens de casos confirmados de tuberculose encontram-se nos estados do Amazonas e Pará, enquanto as menores estão em Rondônia, Acre, Amapá, Tocantins e Roraima. 
Figura 3 Mostra a porcentagem de casos confirmados de tuberculose entre 2013 e 2017 na região Norte do Brasil.

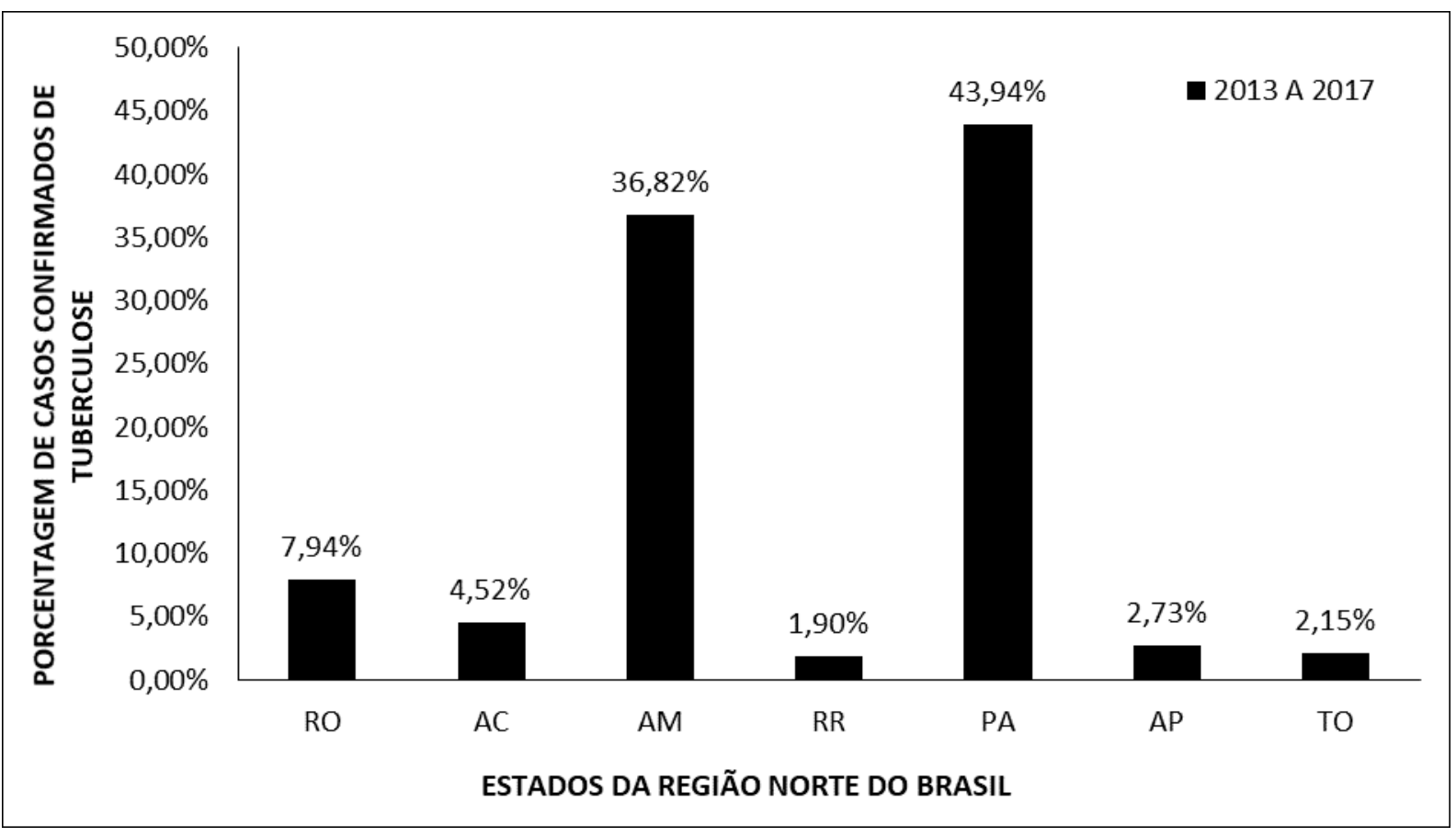

A figura 4 mostra a porcentagem de casos confirmados de tuberculose no estado do Amapá no Brasil entre 2013 e 2017. Entre os anos de 2013 a 2015 houve pouca diferença no aumento e diminuição de casos confirmados, de 2015 para 2016 ocorreu um aumento na porcentagem, e em 2017 manteve-se próximo ao número porcentual próximo do ano de 2016. 
Figura 4 Mostra a porcentagem de casos confirmados de tuberculose no estado do Amapá no Brasil entre 2013 e 2017.

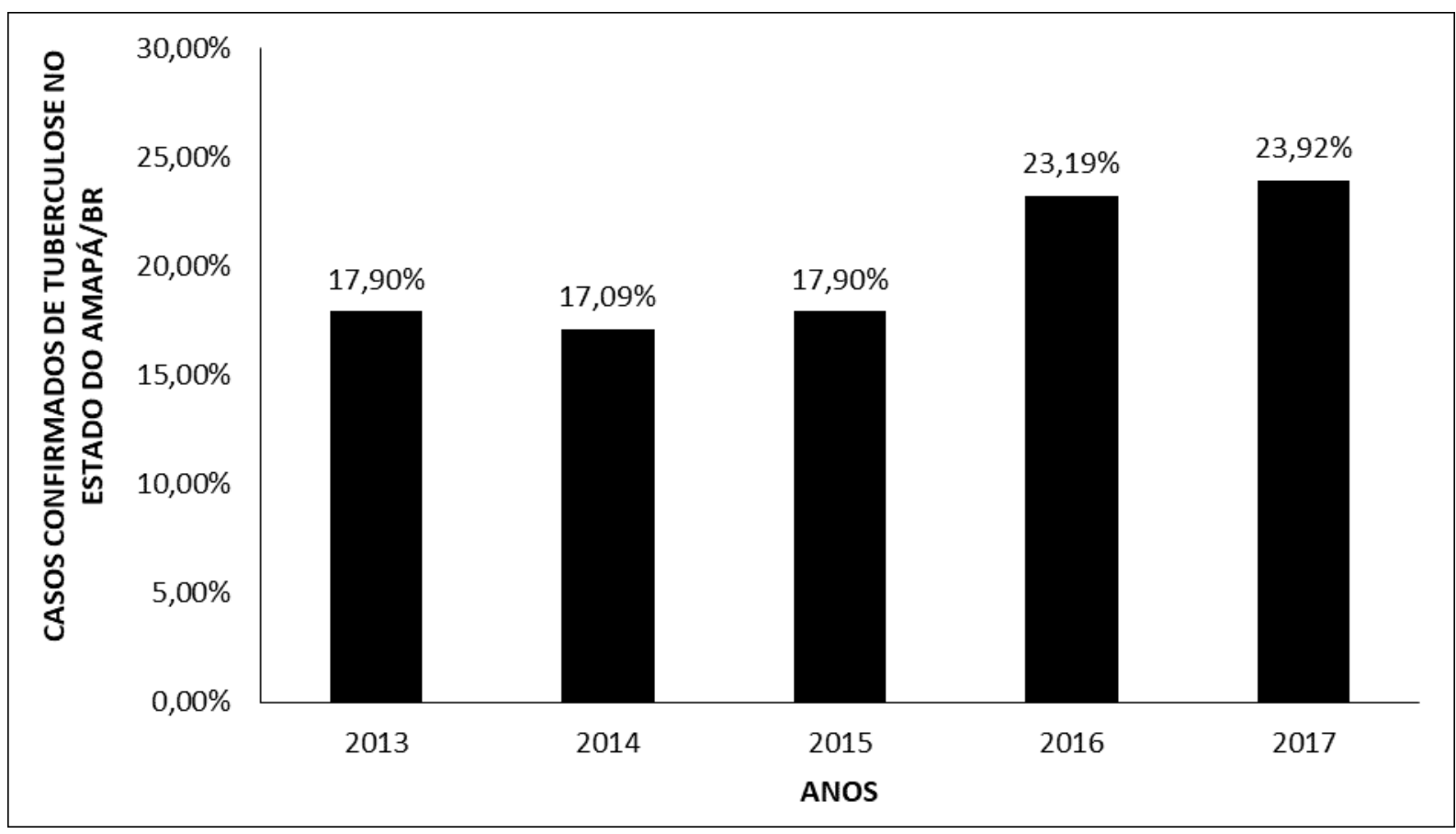

A figura 5 mostra a porcentagem de casos confirmados de tuberculose na cidade de Macapá/AP entre os anos de 2013 e 2017. De 2013 a 2014 a porcentagem de casos confirmados teve uma pequena diminuição, e a partir de 2015 até 2017 apresentou um crescimento. 
Figura 5 Mostra a porcentagem de casos confirmados de tuberculose na cidade de Macapá/AP entre os anos de 2013 e 2017.

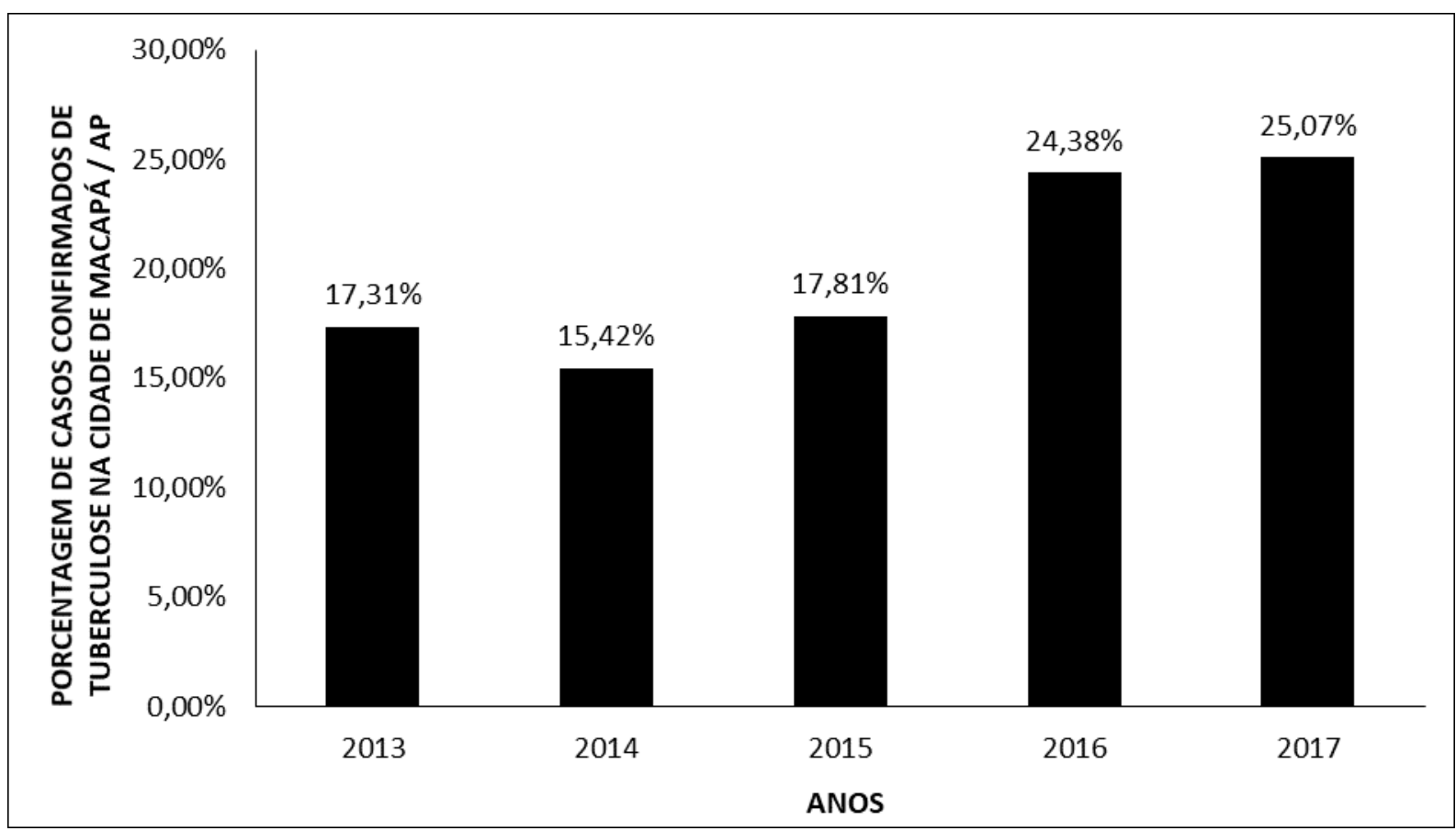

A figura 6 mostra a porcentagem de casos confirmados de tuberculose de acordo com faixa etária na cidade de Macapá/AP entre 2013 e 2017. Nota-se que as maiores porcentagens de casos confirmados estão na faixa etária de 20-39 e 40-59 e as menores estão nas de 60-79, 1-19, 80-+ e $<1$ ano. 
Figura 6 Mostra a porcentagem de casos confirmados de tuberculose de acordo com faixa etária na cidade de Macapá/AP entre 2013 e 2017.

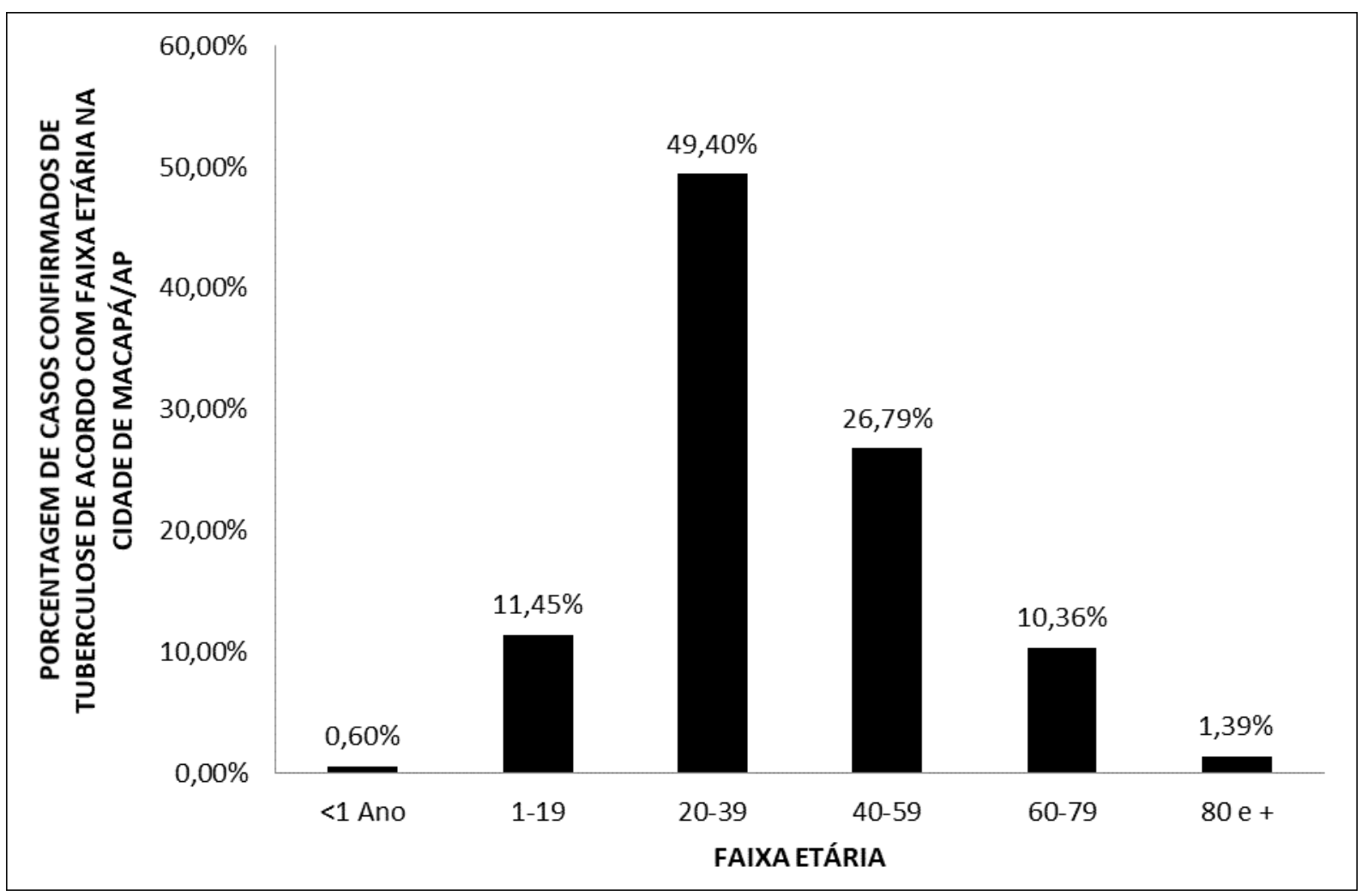

A figura 7 mostra a porcentagem de casos confirmados de tuberculose de acordo com escolaridade na cidade de Macapá/AP entre 2013 e 2017. Nota-se que a maior porcentagem de casos confirmados ocorre em indivíduos com ensino médio completo, seguido por ensino fundamental completo e ensino superior. A menor porcentagem de casos ocorre em sujeitos com $4^{2}$ série do ensino fundamental completo. A taxa de casos ignorados ou em branco é superior a todas as outras. 
Figura 7 Mostra a porcentagem de casos confirmados de tuberculose de acordo com escolaridade na cidade de Macapá/AP entre 2013 e 2017.

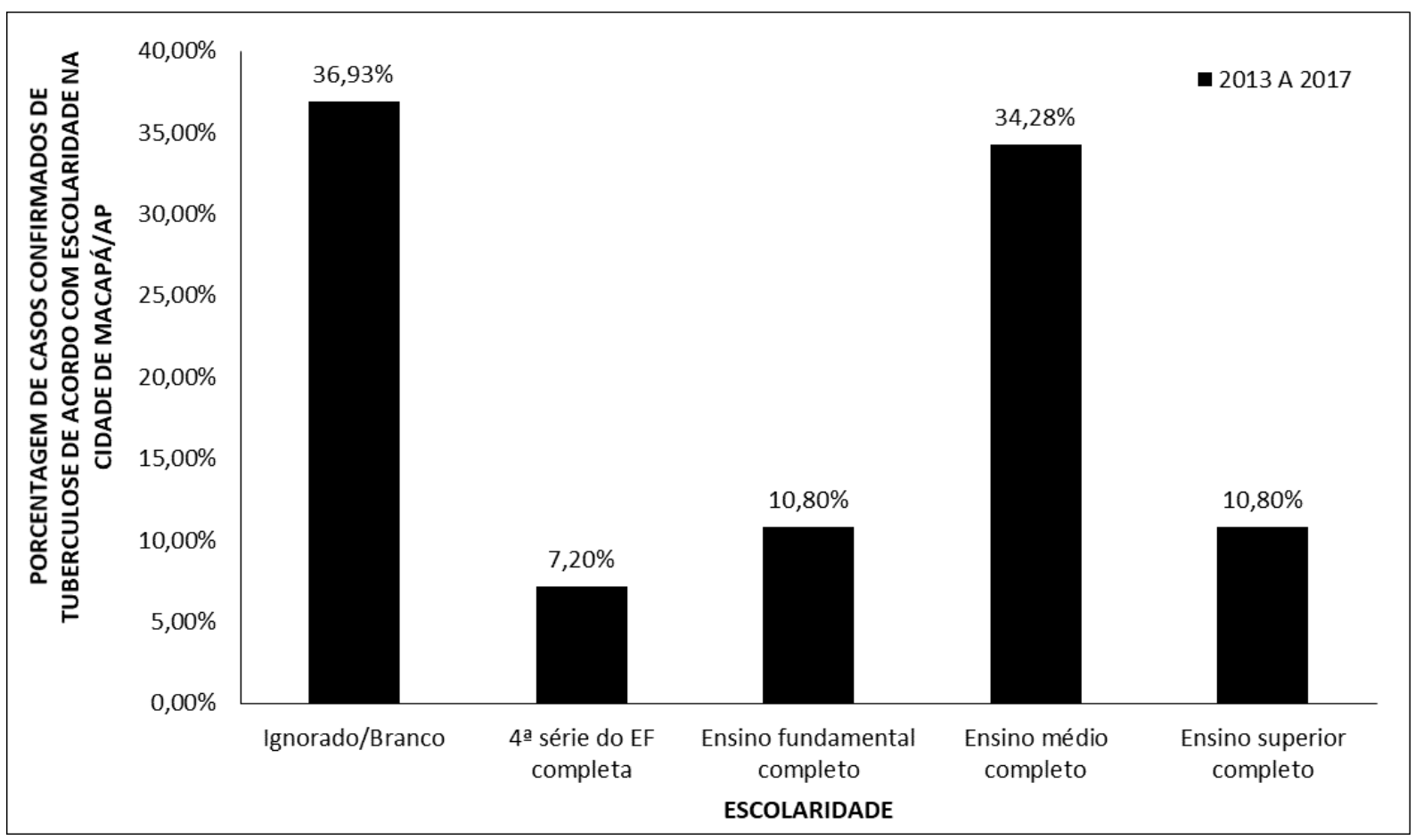

\section{DISCUSSÃO}

Um dos principais fatores que podem ser correlatos ao aumento dos casos de tuberculose (TB) no Brasil, em 2016 e 2017 (Figura 1), é a coinfecção pelo vírus da imunodeficiência humana (HIV) (BRASIL, 2017a). Essa coinfecção é responsável por fragilizar o sistema imune do indivíduo, facilitando a entrada de organismos oportunistas, como é o caso do Bacilo de Koch, causador da tuberculose. Comparado ao ano de 2015, em 2016 houve um aumento de 4\% no número de novos infectados por HIV (38.090 casos) no Brasil. Ainda em 2016, cerca de 76\% dos novos casos de TB foram testados para uma possível coinfecção por HIV e, dentre eles, 12\% (6501 pessoas) estavam coinfectadas, denotando parcela significativa do valor (BRASIL, 2017b). Além disso, no geral, o alcoolismo, uso de drogas, condições sociosespaciais (aglomerações urbanas), socioeconômicas e o estado nutricional do indivíduo também podem representar fatores atrelados à ocorrência de TB (VALENTE et al., 2019). 
A região Sudeste (Figura 2), apresenta a maior população por regiões e muitos de seus municípios possuem alto Índice de Desenvolvimento Humano (IDH). Todavia, também apresenta elevada taxa de pessoas em situação de vulnerabilidade social, caracterizada por vários fatores, sendo a escassa cobertura da atenção primária a saúde um dos principais. A ampla desigualdade econômica reflete no acesso à saúde, uma vez que os mais pobres não conseguem recorrer a hospitais privados para tratamento, dependendo amplamente dos serviços de atenção primária (RANZANI et al., 2018).

O estado do Amazonas (Figura 3) possui abrangente população indígena mais suscetível ao agravamento de doenças ocasionadas por microrganismos, devido a condições de saúde precárias e desfavoráveis (principalmente em sua porção da fronteira internacional que compreende um total de 9.058 quilômetros do Acre ao Amapá). Devido a grande movimentação no espaço fronteiriço ocorre facilidade na transmissão interpessoal de tuberculose (BELO et al., 2013). No Pará, mediante aumento da quantidade de regiões carentes e com o crescimento denso e desordenado de regiões urbanizadas (escassez de saneamento, água e eletricidade), houve também aumento da incidência de casos de tuberculose, reforçando a influência de fatores econômicos e socioespaciais no elevado número de casos da doença (LIMA et al., 2017).

No Amapá (Figura 4), o órgão responsável pelo serviço de atendimento especializado a doenças infecciosas, entre elas a tuberculose, é o Centro de Referência em Doenças Tropicais (CRDT), que também agrega o Centro de Testagem e Aconselhamento (CTA) e Assistência Domiciliar Terapêutica (ADT). O aumento da cobertura do sistema de testagem e diagnóstico em alguns municípios do estado pode ter sido o responsável por revelar casos antes não detectados, os quais ficavam à margem dos dados disponíveis (AMAPÁ, 2016).

A capital Macapá (Figura 5) é uma das cinco cidades em que é possível realizar a testagem para tuberculose no estado do Amapá (AMAPÁ, 2016). Em 2016, abrangeu 151 dos 230 novos casos de tuberculose do estado e, desses casos, 16\% representavam coinfecções por HIV (BRASIL, 2017b). Em 2017, junto a Roraima, 
Macapá apresentou uma das maiores taxas de detecção de AIDS (Síndrome da Imunodeficiência Adquirida), sendo ela de 29,8 por 100 mil habitantes. Dessa forma, os casos de coinfecção, por conseguinte, tendem a aumentar, elevando o número total de casos de tuberculose (BRASIL, 2018).

Analisando o panorama da tuberculose no Brasil, a faixa etária de maior acometimento em mulheres é de 20-39 anos. Já em homens, a faixa etária é de 40-59 anos. Os homens representam a maioria da incidência no país. Em 2012 sua incidência era de 50,2 para 100 mil habitantes, um valor mais de 2 vezes superior ao do sexo feminino (BRASIL, 2017a). Dessa forma, ao analisarmos os gráficos da figura 6 , notamos que Macapá corresponde à média da faixa etária nacional para tuberculose. Na fase adulta, além de enfrentarem maiores aglomerações de pessoas por conta do trabalho, o que aumenta a transmissão (LIMA et al., 2017), a vacina BCG não surte efeito (CARVALHO et al., 2018). Isso pode tornar adultos mais suscetíveis elevando o número de casos nesta faixa de idade (SILVA et al., 2020).

Em âmbito nacional, o perfil para infecção por tuberculose mostra-se diretamente atrelado a homens, principalmente os que possuem idade economicamente ativa e nível reduzido de escolaridade. Eles têm pouco acesso a informações sobre a tuberculose no decorrer da vida e baixo entendimento sobre a patologia e suas consequências orgânicas. A segregação socioespacial e baixa renda também fazem parte desse perfil. Todos esses fatores, combinados, acabam por acarretar o abrangente abandono do tratamento para TB. Em vista dessa interrupção terapêutica, o indivíduo pode favorecer a seleção de bactérias, levando à resistência medicamentosa e, consequentemente, o possível agravamento da doença (RODRIGUES e MELLO, 2018).

\section{CONCLUSÃO}

As coinfecções assim como o alcoolismo, uso de drogas, aglomerações urbanas e o estado nutricional do indivíduo também podem representar fatores que aumentam os casos de tuberculose. A vulnerabilidade social e a pouca atenção primária a saúde também favorecem o aumento desses números. Ter um Centro de Referência em 
Doenças Tropicais (CRDT), que também agrega o Centro de Testagem e Aconselhamento (CTA) e Assistência Domiciliar Terapêutica (ADT) auxilia na detecção de casos agilizando e controlando o tratamento de pacientes. Homens na faixa etária de trabalho são mais acometidos por conta da sua necessária interação social para o trabalho.

\section{REFERENCIAS}

AMAPÁ. Plano Estadual de Saúde 2016-2019. Macapá AP: Governo do Estado do Amapá: 244 p. 2016.

BELO, E. N. et al. Tuberculose nos municípios amazonenses da fronteira BrasilColômbia-Venezuela: situação epidemiológica e fatores associados ao abandono Rev Panam Salud Publica, v. 24, n. 5, p. 321-329, 2013.

BRASIL. Boletim Epidemiológico. Brasilia DF: Ministério da Saúde. 40: 12 p. 2017 a. Boletim Epidemiológico HIV AIDS. Brasilia DF: Ministério da Saúde: 64 p. 2017b.

Boletim Epidemiológico 11. Brasilia DF: Ministério da Saúde. 49: 18 p. 2018.

Manual de recomendações para o controle da tuberculose no Brasil. Brasilia DF: Ministério da Saúde: 366 p. 2019.

CARVALHO, A. C. C. et al. Aspectos epidemiológicos, manifestações clínicas e prevenção da tuberculose pediátrica sob a perspectiva da estratégia End TBv. J Bras Pneumol., v. 44, n. 2, p. 134-144, 2018.

KOZAKEVICH, G. V.; SILVA, R. M. D. Tuberculose: Revisão de Literatura. Arq. Catarin Med. , v. 44, n. 4, p. 34-47, 2015.

LIMA, S. S. et al. Análise espacial da tuberculose em Belém, estado do Pará, Brasil. v. Rev Pan-Amaz Saude, n. 2, p. 57-65, 2017. 
MACEDO, J. L. et al. Perfil epidemiológico da tuberculose em um Município do Maranhão. ReonFacema, v. 3, n. 4, p. 699-705, 2017.

RANZANI, O. T. et al. Quem são os pacientes com tuberculose diagnosticados no pronto-socorro? Uma análise dos desfechos do tratamento no estado de São Paulo, Brasil. J Bras Pneumol, v. 44, n. 2, p. 125-133, 2018.

RODRIGUES, M. W.; MELLO, A. G. N. C. Tuberculose e escolaridade: Uma revisão de literatura. Revista Internacional de Apoyo a la Inclusion, Logopedia, Sociedad y Multiculturalidad, v. 4, n. 2, p. 01-12, 2018.

SILVA, F. T. et al. Número de casos confirmados de tuberculose no Brasil entre 2007 e 2016. Revista Científica Multidisciplinar Núcleo do Conhecimento, v. 4, n. 8, p. 94-104, 2020.

SILVA, M. E. N. D. et al. Aspectos gerais da tuberculose: uma atualização sobre o agente etiológico e o tratamento. RBAC, v. 50, n. 3, p. 228-232, 2018.

VALENTE, B. C. et al. A tuberculose e seus fatores associados em um município da região metropolitana do Rio de Janeiro. , v. 22, e190027, . Rev Bras Epidemiol, v. 22, p. 1 - 12, 2019.

WHO. Global Tuberculosis Report. Executive Summary. 2019. Disponível em: < https://www.who.int/tb/publications/global_report/GraphicExecutiveSummary.pdf?ua= 1\&ua=1 >. Acesso em: 03 maio 2020.

\section{APÊNDICE - REFERÊNCIAS DE NOTA DE RODAPÉ}

7. http://datasus.saude.gov.br/

8. http://datasus.saude.gov.br/

Enviado: Maio, 2020.

Aprovado: Maio, 2020. 